\title{
Síndrome de hiperestimulación ovárica severo: Informe de caso y revisión de literatura
}

\author{
Severe Ovarian Hyper Stimulation Syndrome: Case Report and \\ Literature Review
}

\section{Síndrome de hiperestimulação ovárica grave: Relato de caso e revisão da literatura}

\author{
Zulma Esperanza Urbina-Contreras, MD., Esp. * \\ Sergio Enrique Urbina-Echeverry, MD., Esp. ** \\ Andrés Felipe Lamos-Duarte, MD., Esp. *** \\ Yelson Alejandro Picón-Jaimes, MD. ****
}

\section{Resumen}

Introducción: El síndrome de hiperestimulación ovárica severo es la complicación más grave de la estimulación ovárica durante la inducción de la ovulación. Los hallazgos característicos son la presencia de ovarios aumentados de volumen y ascitis en relación a extravasación de líquido por aumento de la permeabilidad capilar, cuya severidad puede llevar a falla respiratoria, renal, colapso hemodinámico y eventos tromboembólicos. Objetivo: Describir un caso clínico de síndrome de hiperestimulación ovárica, junto con una revisión de la literatura sobre la patología, orientada al diagnóstico y tratamiento óptimo de pacientes con estas características clínicas. Presentación del caso: Paciente de 29 años con antecedente de síndrome de ovario poliquístico que presenta síndrome de hiperestimulación ovárica severo como complicación secundaria a estimulación gonadotrófica. El cuadro clínico mostró ovarios aumentados de tamaño en la evaluación sonográfica; anasarca dada por ascitis y derrames pleurales bilaterales e insuficiencia respiratoria tipo distrés respiratorio del adulto. Se realizó paracentesis y se utilizó ventilación mecánica no invasiva, logrando la expansión pulmonar. Discusión: El análisis de este caso se inició siete días después de la administración de gonadotropina, favorecido por las condiciones previas de la paciente, sin complicaciones letales. Conclusiones: La paracentesis puede constituir una opción terapéutica efectiva en el tratamiento de ascitis con compromiso de la función pulmonar. La ventilación mecánica no invasiva es una estrategia para evitar la intubación en estas pacientes lo cual evita los periodos de sedación exhaustivos y el consiguiente riesgo de broncoaspiración. [Urbina-Contreras ZE, Urbina-Echeverry SE, Lamos-Duarte AF, Picón-Jaimes YA. Síndrome de hiperestimulación ovárica severo: Informe de caso y revisión de literatura. MedUNAB 2017; 20(2): 244-251].

Palabras Clave: Síndrome de Hiperestimulación Ovárica; Inducción de la Ovulación; Fármacos para la Fertilidad Femenina; Gonadotropina Coriónica; Infertilidad Femenina.

\footnotetext{
* Médica, especialista en Medicina Interna e Intensivista. Hospital Universitario Erasmo Meoz, Cúcuta, Norte de Santander, Colombia.

** Médico, especialista en Ginecología y Obstetricia e Intensivista. Hospital Universitario Erasmo Meoz, Cúcuta, Norte de Santander, Colombia.

*** Médico, especialista en Epidemiología, docente Universidad de Boyacá, Tunja, Boyacá, Colombia.

**** Médico. Clínica Chía, Chía, Cundinamarca, Colombia
}

Correspondencia:Yelson Alejandro Picón Jaimes. Carrera 18 No. 113-52, Edificio Puerto Madero, San Patricio, Bogotá, Colombia. Teléfono: +57 3105747525. E-mail: ypicon@unab.edu.co 


\section{Abstract}

Introduction: Severe ovarian hyper stimulation syndrome is the most serious complication of ovarian stimulation during the induction of ovulation. Characteristic findings are the presence of increased ovarian volume and ascites in relation to fluid extravasation due to increased capillary permeability whose severity can lead to respiratory failure, renal failure, and hemodynamic collapse and thromboembolic events. Objective: To describe a clinical case of OHSS, along with a review of the literature on the pathology, aimed at the diagnosis and optimal treatment of patients with these clinical characteristics. Case presentation: A 29-year-old patient with a history of polycystic ovarian syndrome who presented severe Severe ovarian hyper stimulation syndrome as a secondary complication to gonadotrophic stimulation. The clinical profile showed enlarged ovaries in the sonographic test; anasarca due to ascites and bilateral pleural effusions and respiratory failures that belong to a respiratory distress type in this adult patient. Paracentesis was performed and noninvasive mechanical ventilation was used, achieving lung expansion with it. Discussion: The analysis of this case was started seven days after the administration of gonadotropin, favored by the patient's previous conditions, with no lethal complications. Conclusions: Paracentesis may be an effective therapeutic option in the treatment of ascites with compromised lung function. Noninvasive mechanical ventilation is a strategy to avoid intubation in these patients, which avoids the periods of exhaustive sedation and the consequent risk of bronchoaspiration. [Urbina-Contreras ZE, Urbina-Echeverry SE, Lamos-Duarte AF, Picón-Jaimes YA. Severe Ovarian Hyper Stimulation Syndrome: Case Report and Literature Review. MedUNAB 2017; 20(2): 244-251].

Keywords: Ovarian Hyperstimulation Syndrome; Ovulation Induction; Fertility Agents, Female; Chorionic Gonadotropin; Infertility, Female.

\section{Introducción}

El síndrome de hiperestimulación ovárica (SHEO) se caracteriza por el desplazamiento del volumen plasmático intravascular hacia un tercer espacio como respuesta fisiológica exagerada posterior a la administración exógena de Gonadotropina Coriónica Humana (hCG) en los procesos de ovulación inducida para la reproducción asistida. La hCG desencadena una luteinización de los folículos, con la siguiente liberación de mediadores a nivel ovárico, los cuales aumentan la permeabilidad capilar y promueven la angiogénesis (1-3).

El SHEO es una complicación iatrogénica potencialmente mortal en sus estadios más graves, desencadenando disfunción renal, hepática, vascular y respiratoria $(1,4,5)$. Los casos leves se presentan hasta en el $33 \%$ de las pacientes, mientras los graves oscilan entre el 0.1 y el $5 \%$. Esta variabilidad puede deberse a la falta de estudios que busquen medir la real incidencia, así como los diferentes protocolos de manejo que hacen que estos procedimientos

\section{Resumo}

Introdução: O síndrome de hiperestimulação ovárica avançado é a complicação mais grave da estimulação ovariana durante a indução da ovulação. As características achadas são o aumento do volumem dos ovários e ascite em relação ao extravasamento do fluido devido ao aumento da permeabilidade capilar, cuja gravidade pode levar à insuficiência respiratória, insuficiência renal, colapso hemodinâmico e eventos tromboembólicos. Objetivo: Descrever um caso clínico de síndrome de hiperestimulação ovárica avançado, juntamente com uma revisão da literatura sobre a patologia, visando o diagnóstico e tratamento ótimo de pacientes com essas características clínicas. Apresentação do caso: Paciente de 29 anos com história de síndrome de ovário policístico que apresenta síndrome de hiperestimulação ovárica avançado como complicação secundária à estimulação gonadotrófica. O quadro clínico mostrou ovários ampliados na avaliação ultrasonográfica; anasarca devido à ascite e derrames pleurais bilaterais e insuficiência respiratória do tipo insuficiência respiratória do adulto. Foi realizada a paracentese e foi utilizada ventilação mecânica não invasiva, atingindo a expansão pulmonar. Discussão: A análise deste caso começou sete dias após a administração da gonadotrofina, favorecida pelas condições prévias da paciente, sem complicações letais. Conclusões: A paracentese pode constituir uma opção terapêutica efetiva no tratamento de ascite com comprometimento da função pulmonar. A ventilação mecânica não invasiva é uma estratégia para evitar intubação nessas pacientes, o que evita os períodos de sedação exaustiva e o consequente risco de bronco-aspiração. [Urbina-Contreras ZE, Urbina-Echeverry SE, Lamos-Duarte AF, Picón-Jaimes YA. Síndrome de hiperestimulação ovárica grave: Relato de caso e revisão da literatura. MedUNAB 2017; 20(2): 244-251].

Palavras-chave: Síndrome de Hiperestimulação Ovariana; Indução da Ovulação; Fármacos para a Fertilidade Feminina; Gonadotropina Coriônica; Infertilidade Feminina.

sean diferentes a lo largo de la geografía mundial $(1,4,6)$. La mortalidad reportada es de 1 por cada 50,000 pacientes $(7,8)$.

Los péptidos vasoactivos liberados por las células de la granulosa en el ovario hiperestimulado, incrementan la permeabilidad de los vasos sanguíneos, generando así flujo hacia las cavidades peritoneal y torácica, lo cual da como resultado depleción de volumen intravascular y hemoconcentración de las células plasmáticas $(4,6)$. Uno de los mediadores implicados en la fisiopatología de este síndrome es el factor de crecimiento derivado del endotelio vascular, el cual se libera en presencia de hCG y su concentración plasmática es directamente proporcional a la severidad del cuadro clínico (4). Otras moléculas participes de esta cascada de permeabilidad son la angiotensina II, el factor de crecimiento similar a la insulina y la interleucina 6 $(4,5)$.

Con los constantes desarrollos en tema de fertilidad y reproducción asistida aumenta la atención a estas pacientes en los servicios de emergencia, por lo cual se presenta un caso, acompañado de revisión de la literatura médica. 


\section{Presentación del caso}

Paciente de 29 años con diagnóstico de infertilidad primaria secundaria a síndrome de ovario poliquístico, en tratamiento con estimulación ovárica controlada (EOC). Recibió inducción de ovulación con 5,000 UI de hCG y 36 horas después se obtuvo 10 oocitos por aspiración. Ingresa al servicio de urgencias cuatro días después, diferida de un hospital de baja complejidad por cuadro que inició el día siguiente a la aspiración con dolor abdominal y emésis que evolucionó hasta aparecer insuficiencia renal aguda, ascitis, hemoconcentración (hematocrito de 45\%) y trastorno hidroelectrolítico (hiponatremia e hipokalemia). La paciente es llevada a Unidad de Cuidados Intensivos (UCI) donde el estudio de ecografía abdominal reveló abundante liquido libre en cavidad, por lo cual, se realizó paracentesis evacuadora con drenaje de $2,000 \mathrm{ml}$ de líquido ascítico de aspecto serohemático ya que en la institución no se contaba con personal entrenado en la realización de colpocentesis guiada por ecografía. Paraclínicos adicionales demostraron acidosis metabólica, hiponatremia e hipokalemia y leucocitosis (Tabla 1).

Tabla 1. Paraclínicos durante la hospitalización de la paciente en la unidad de cuidados intensivos.

\begin{tabular}{|c|c|c|c|c|}
\hline Estancia & Función renal & $\begin{array}{c}\text { Perfil } \\
\text { Hepático u/l }\end{array}$ & Perfil hemático & Gasometría arterial \\
\hline Ingreso & $\begin{array}{l}\text { Cr: } 1 \mathrm{mg} / \mathrm{dl} \\
\text { BUN:12 mg/dl }\end{array}$ & Sin reporte & $\begin{array}{l}\text { Hcto: } 45 \% \\
\text { Leucos:22,400/ml }\end{array}$ & $\begin{array}{l}\mathrm{PH}: 7.3 \\
\text { PO2: } 75 \mathrm{mmHg} \\
\text { PCO2: } 27 \mathrm{mmHg} \\
\text { HCO3: } 15 \mathrm{mEg} / \mathrm{L}\end{array}$ \\
\hline Día 2 & Sin reporte & $\begin{array}{l}\text { AST: } 42 \\
\text { ALT: } 37\end{array}$ & Sin reporte & Sin reporte \\
\hline Día 3 & $\begin{array}{l}\text { Cr: } 1 \mathrm{mg} / \mathrm{dl} \\
\text { BUN:23 mg/dl }\end{array}$ & Sin reporte & $\begin{array}{l}\text { Hcto: } 35 \% \\
\text { Leucos: } 31,000 / \mathrm{ml}\end{array}$ & $\begin{array}{l}\text { PO2: } 55 \mathrm{mmHg} \\
\text { PCO2: } 27 \mathrm{mmHg} \\
\text { HCO3: } 20 \mathrm{mEq} / \mathrm{L}\end{array}$ \\
\hline Día 4 & $\begin{array}{l}\text { Cr: } 0.77 \mathrm{mg} / \mathrm{dl} \\
\text { BUN:23 mg/dl }\end{array}$ & Sin reporte & Leucos: $17,000 / m l$ & Sin reporte \\
\hline Día 5 & $\begin{array}{l}\text { Cr: } 0.84 \mathrm{mg} / \mathrm{dl} \\
\text { BUN:23 mg/dl }\end{array}$ & Sin reporte & $\begin{array}{l}\text { Hcto: } 33 \% \\
\text { Leucos: } 15,430 / \mathrm{ml}\end{array}$ & $\begin{array}{l}\mathrm{PH}: 7,4 \\
\text { PO2: } 65 \mathrm{mmHg} \\
\text { PCO2: } 27 \mathrm{mmHg} \\
\text { HCO3: } 24 \mathrm{mEq} / \mathrm{L}\end{array}$ \\
\hline Día 6 & Sin reporte & $\begin{array}{l}\text { AST: } 45 \\
\text { ALT: } 50\end{array}$ & Leucos: 12,800/ml & Sin reporte \\
\hline Día 7 & $\begin{array}{l}\mathrm{Cr}: 0.74 \mathrm{mg} / \mathrm{dl} \\
\text { BUN: } 12.19 \\
\mathrm{mg} / \mathrm{dl}\end{array}$ & Sin reporte & Leucos: $11,490 / \mathrm{ml}$ & Sin reporte \\
\hline Día 8 & $\begin{array}{l}\text { Cr: } 0.9 \\
\text { BUN: } 16 \mathrm{mg} / \mathrm{dl}\end{array}$ & Sin reporte & Leucos: 12,000/ml & Sin reporte \\
\hline Día 9 & Sin reporte & $\begin{array}{l}\text { AST: } 50 \\
\text { ALT: } 55\end{array}$ & Sin reporte & Sin reporte \\
\hline Día 10 & Sin reporte & Sin reporte & $\begin{array}{l}\text { Hcto: } 32 \% \\
\text { Leucos: } 9,700 / \mathrm{ml}\end{array}$ & Sin reporte \\
\hline Día 11 & $\begin{array}{l}\mathrm{Cr}: 0.7 \mathrm{mg} / \mathrm{dl} \\
\text { BUN: } 12.13 \\
\mathrm{mg} / \mathrm{dl}\end{array}$ & Sin reporte & Sin reporte & $\begin{array}{l}\mathrm{PH}: 7,4 \\
\text { PO2: } 85 \mathrm{mmHg} \\
\text { PCO2: } 34 \mathrm{mmHg} \\
\text { HCO3: } 24 \mathrm{mEq} / \mathrm{L}\end{array}$ \\
\hline Día 14 & $\begin{array}{l}\mathrm{Cr}: 0.7 \mathrm{mg} / \mathrm{dl} \\
\text { BUN: } 12.11 \\
\mathrm{mg} / \mathrm{dl}\end{array}$ & $\begin{array}{l}\text { AST: } 32 \\
\text { ALT: } 30\end{array}$ & $\begin{array}{l}\text { Hcto: } 33 \% \\
\text { Leucos: } 6,700 / \mathrm{ml}\end{array}$ & $\begin{array}{l}\text { PH: } 7,4 \\
\text { PO2: } 87 \mathrm{mmHg} \\
\text { PCO2: } 27 \mathrm{mmHg} \\
\text { HCO3: } 19 \mathrm{mEq} / \mathrm{L}\end{array}$ \\
\hline
\end{tabular}

${ }^{*} \mathrm{Cr}$ : creatinina, BUN: Nitrógeno ureico en sangre, Hcto: hematocrito, Leucos: Leucocitos, AST: aspartato aminotransferasa, ALT: alanina aminotransferasa, $\mathrm{PH}$ : concentración de iones hidrógeno $[\mathrm{H}]+$, PO2: Presión parcial de oxígeno, PCO2: presión parcial de dióxido de carbono, HCO3: bicarbonato.

Fuente: Elaboración de los autores. 
El manejo inicial incluyo bloqueadores del nodo AV para la taquicardia refleja, diuréticos de ASA, orientados por el gasto urinario bajo y reposición isovolúmetrica. Al tercer día de estancia, se observó aumento significativo de perímetro abdominal y deterioro respiratorio con $\mathrm{PaO} 2$ de $55 \mathrm{mmHg}$ y $\mathrm{PCO} 2$ de $27 \mathrm{mmHg}$, requiriendo de Ventilación Mecánica No Invasiva (VMNI). Al cuarto día mostró tendencia a la mejoría, con mecánica ventilatoria acorde, gasimetría con disfunción leve en oxigenación, equilibrio ácido base y electrolítico. En el séptimo día, se reportó estradiol en rangos de severidad en 3,000 UI, sin cambios importantes en la evolución clínica, hasta el décimo día, cuando se observó mejoría hacia metas de estabilización propuestas, mostrando solo elevación leve de las transaminasas.

Finalmente, se dio egreso el día 14 con recomendaciones y seguimiento en habitación hospitalaria. Se obtuvo consentimiento informado por parte de la paciente para el uso de la información clínica contenida en su expediente, así como el uso de los datos de sus paraclínicos.

\section{Discusión y revisión de la literatura}

El síndrome de hiperestimulación ovárica severo es una respuesta iatrogénica secundaria a la inducción farmacológica de la ovulación en el tratamiento de la infertilidad femenina. Aunque en ocasiones se puede presentar en embarazos espontáneos en pacientes con hipersensibilidad a la HCG endógena $(3,8)$.

El Factor de crecimiento endotelial vascular, las citokinas proinflamatorias (IL $-2, \mathrm{IL}-6, \mathrm{IL}-8)$, el factor de necrosis tumoral alfa y el sistema renina-angiotensina ovario, los cuales son activados por las gonadotrofinas, llevan a un estado de permeabilidad vascular y acumulación de líquidos a nivel extravascular $(9,10)$.

Estas alteraciones del endotelio y mesotelio determinan las características clínicas de las formas severas de la hiperestimulación ovárica, que incluyen ascitis, derrames pleurales, edema pulmonar, hipovolemia e insuficiencia renal pre renal y eventos tromboembólicos. La paciente de este caso presentó insuficiencia respiratoria, con derrames pleurales y edema pulmonar. El trabajo respiratorio y las alteraciones gasimétricas mostraron mejoría progresiva en relación con el uso de la ventilación mecánica no invasiva. No fue requerida la toracentesis ni la toracostomia para el manejo del derrame pleural secundario. La ventilación mecánica no invasiva, de manera intensiva durante la hospitalización en la UCI, disminuye el riesgo de neumonía relacionada con el ventilador y resolución de derrames pleurales y edema pulmonar, como una estrategia no consensuada para estas indicaciones (9).

Los ajustes terapéuticos durante la inducción de ovulación en pacientes con factores relacionados con un mayor riesgo para inducir SHEO deben ser considerados para prevenir esta complicación (9).

Este caso de síndrome de hiperestimulación ovárica fue de temporalidad temprana, ya que ocurrió en los siete días posteriores a la administración exógena de GCH. En la literatura médica, se describe que este cuadro es autolimitado (si no hay gestación) y por ende es de menor severidad (5).

No obstante, por tratarse de una paciente joven con antecedente de infertilidad posiblemente secundaria a síndrome de ovario poliquístico, era poco probable la aparición de complicaciones potencialmente letales (fracaso renal, falla hepática, shock hipovolémico, fenómenos tromboembolicos y SDRA). Las complicaciones vitales que sucedieron en esta paciente fueron: fracaso renal agudo y hemorragia por rotura folicular con anemia. Así mismo, el fenómeno de tercer espacio relacionado con la ascitis severa se asoció con hidrotórax, producto de la transferencia del líquido abdominal a la cavidad torácica posiblemente a través del conducto torácico. Respecto a las medidas evacuadoras, la paracentesis normalmente fue efectiva en la resolución indirecta del hidrotórax y la toracentesis debe ser reservada para los casos con derrames bilaterales o severos. La paracentesis guiada por ecografía se indicó en 4 oportunidades por ascitis a tensión con función pulmonar comprometida y oliguria/anuria que no mejoró con el manejo de fluidos apropiado. Se apreció elevación de transaminasas, situación frecuente en estas pacientes, su etiología es desconocida y no hay un tratamiento dirigido, sino que su resolución va acorde al restablecimiento de la homeostasis de los diferentes sistemas.

Los factores de riesgo para desarrollar el SHEO son: edad menor de 35 años, índice de masa corporal bajo 18.5, síndrome de ovario poliquístico, embarazo, incremento abrupto de los niveles de estrógenos por encima de $4,000 \mathrm{pg} / \mathrm{mL}$, desarrollo de más de ocho folículos en un ovario, mutaciones de la hormona folículo estimulante y antecedente de síndrome de hiperestimulación ovárica $(7,11)$.

\section{Fisiopatología}

Diversos factores vasoactivos reguladores del equilibrio mineralocorticoide y agentes pro-inflamatorios han sido descritos como el sustrato fisiopatológico de esta enfermedad, entre los que se destacan por orden de importancia: el factor de crecimiento endotelial vascular, sistema renina- angiotensina, algunas prostaglandinas, el factor de necrosis tumoral alfa, el factor de crecimiento similar a la insulina tipo $1 \mathrm{y}$ el factor de crecimiento epidérmico $(5,12)$.

En este contexto, las células de la granulosa del ovario producen factor de crecimiento endotelial vascular (FCEV) secundaria a la administración de gonadotropina coriónica, 
el cual es una de las moléculas de actividad vasoactiva, fuertemente relacionada en la patogenia del síndrome de hiperestimulación ovárica. Esta sustancia es 50,000 veces más potente que la histamina para generar vasodilatación, esto explicaría los fenómenos de tercer espacio relacionados a la alta permeabilidad de los lechos vasculares. Se distinguen dos tipos de factores de crecimiento endotelial vascular, el FCEV-1 que interviene en las uniones intercelulares del endotelio vascular y el FCEV-2 que se encarga de la regulación de la permeabilidad, angiogénesis y vasculogénesis $(5,13)$.

Cuando hay hiperestimulación ovárica, la liberación de FCEV-1 y 2 aumenta provocando una pérdida de la función homeostática, lo cual da como resultado la excesiva permeabilidad vascular que lleva finalmente a disfunción en distintos sistemas corporales (5).

Así mismo, el fenómeno "shift" desencadenado en los compartimientos de los diferentes líquidos corporales, termina en una condición resultante descrita en la literatura, como lo es la hipovolemia hiponatrémica. Consecuentemente, la integridad de los diferentes sistemas es afectada, ocasionando fracaso renal agudo por causas prerrenales, progresando hasta una condición de necrosis tubular aguda por hipoxia/isquemia $(14,15)$.

Teniendo en cuenta que esta entidad varía en grados de severidad, es necesario comprender que, la hemocentración asociada a deshidratación conduce a un aumento de la celularidad plasmática, y de esta manera, a procesos trombóticos locales $(8,16)$ (Figura 1).

\section{Manifestaciones clínicas y criterios diagnósticos}

El SHEO leve se caracteriza por la aparición de dolor en hemiabdomen inferior, náuseas, vómitos, dispepsia y diarrea, que aparece 48 horas luego de la aplicación de la gonadotropina coriónica humana (3).

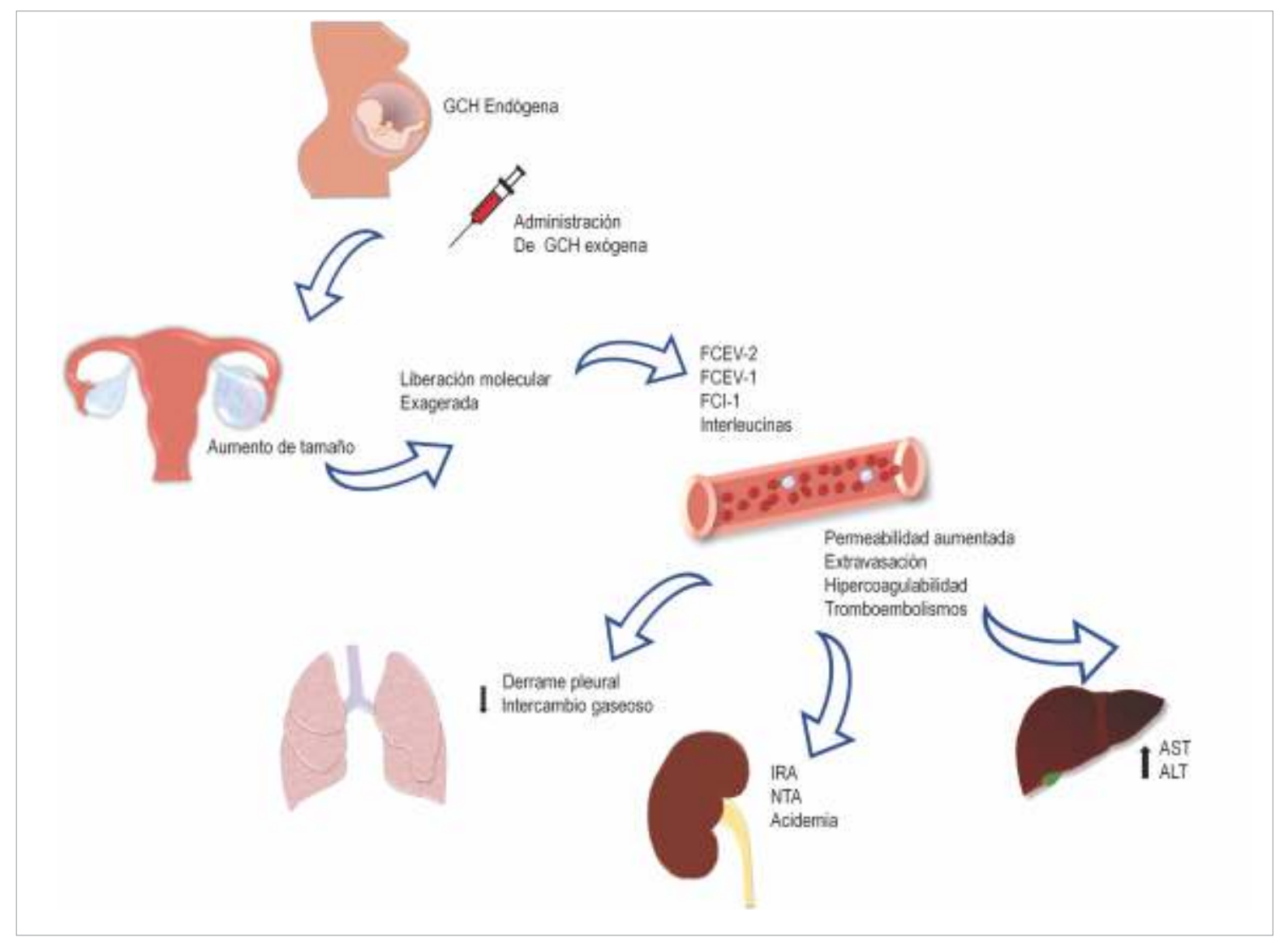

*GCH: Gonadotropina coriónica humana; FCEV-1 y 2: Factor de crecimiento derivado del endotelio vascular 1 y 2; FCl-1: Factor de crecimiento insulínico tipo 1; IRA: Insuficiencia renal aguda; NTA: Necrosis tubular aguda; AST: Aspartato aminotransferasa; ALT: Alanina aminotransferasa.

Fuente: Elaboración de los autores.

Figura 1. Fisiopatología del síndrome de hiperestimulación ovárica 
Los cuadros graves además presentan hipotensión o hipertensión refleja, hipovolemia, desórdenes electrolíticos y síndrome edematoso con afectación de múltiples órganos por la aparición de un tercer espacio (7). Los criterios para la clasificación de la gravedad del SHEO no se han establecido por consenso de especialistas, por esta razón se proponen criterios propios (Tabla 2).

\section{Tratamiento}

El pilar de tratamiento en estas pacientes es de soporte, para contrarrestar los efectos nocivos de la excesiva permeabilidad vascular $(8,15)$. Debe hospitalizarse a pacientes con vómito incoercible, dolor abdominal severo, disnea, oliguria y paraclínicos que hagan sospechar un
SHEO moderado o severo. El manejo debe tener un orden de medidas a seguir:

- Reposición hídrica con expansores de volumen, preferiblemente soluciones cristaloides al medio para mantener una diuresis media de entre 30 y $50 \mathrm{ml} / \mathrm{h}(15,16)$.

- Evaluar la necesidad de realizar paracentesis evacuadoras por técnica convencional o guiada por ultrasonografía (ajustar la reposición hídrica con cristaloides o coloides de elección teniendo en cuenta si hay indicación). Es igualmente valida la colpocentesis como medida evacuadora para el líquido extravasado $(8,15,17)$.

- Evaluar necesidad de toracentesis si existe indicación $(1,5,8)$.

- Plantear esquema con diuréticos de ASA para modular fenómeno de tercer espacio o escenario de paciente oligoanurico:

Tabla 2. Criterios clínicos y paraclínicos para clasificar el SHEO

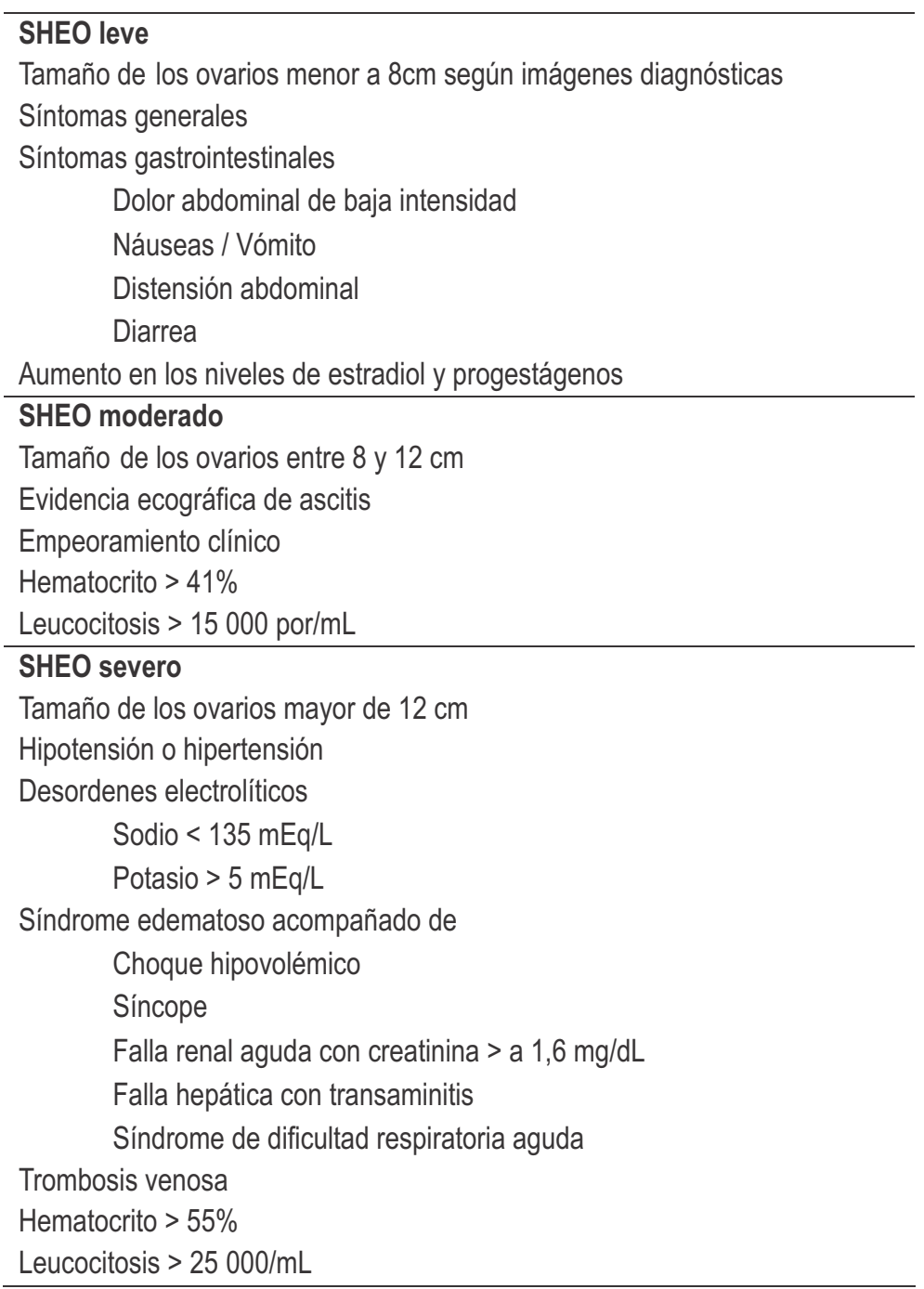

Fuente: Elaboración de los autores, basado en la clasificación del Instituto Nacional para la Excelencia Clínica (NICE) de Inglaterra (2004) 
- Furosemida en infusión.

- Furosemida por horario $20 \mathrm{mg}$ cada 8 horas.

- Dopamina infusión (1-3 ug/kg/min) $(8,14)$.

- Mantener monitoreo hemodinámico y ventilatorio, teniendo en cuenta la gravedad y necesidad del cuadro clínico $(8,14,18)$.

- Iniciar esquema tromboprofiláctico, preferiblemente heparinas de bajo peso molecular, tipo enoxaparina, a dosis estándar desde el primer trimestre del embarazo (8).

- Continuar control diario de paraclínicos dirigidos a la vigilancia de la hemoconcentración, disturbios acidobase, función renal y gasto urinario $(3,15)$ (Figura 2).

\section{Prevención}

\section{a) Prevención primaria}

Se conoce que la aparición del SHEO está ligada al uso de hCG, por ende, si no se administra se podría evitar complicaciones; otras medidas incluyen: identificar factores de riesgo, uso de dosis bajas de gonadotrofinas, maduración con GnRH u hormona luteinizante recombinante (19).

\section{b) Prevención secundaria}

Son todas aquellas medidas que buscan disminuir la aparición del SHEO, cuando se decide aplicar la hCG, entre ellas están: la disminución de dosis utilizada de hCG de
10,000 UI a 3,000-5,000UI; demorar la administración de hCG si se identifican niveles de estradiol mayores a 3,000 $\mathrm{pg} / \mathrm{ml}$ o maduración de más de 20 folículos; aplicación de albúmina profiláctica el día de la aspiración con la finalidad de aumentar la presión oncótica intravascular y disminuir la extravasación de plasma; congelación de embriones retrasando el embarazo, evitando el efecto de la hCG endógena $(16,19)$. En la actualidad el uso de agonistas dopaminérgicos, como la cabergolina son recomendados (8). También es justificable considerar los beneficios del uso de antiplaquetarios en pacientes con antecedentes de fenómenos trombóticos durante la terapia de estimulación ovárica controlada o durante el evento adverso según el caso. No obstante, existe evidencia grado A en el empleo de metformina para disminuir el riesgo significativamente de SHEO en pacientes que tiene como antecedente síndrome de ovario poliquístico $(8,13)$.

\section{c) Prevención terciaria}

Incluye las medidas presentadas en el tratamiento con la finalidad de evitar complicaciones y secuelas en las pacientes que desarrollan SHEO.

\section{Conclusiones}

El SHO es una entidad cuya prevalencia no está esclarecida, sin embargo, se estima que hasta un 5\% de los casos que se

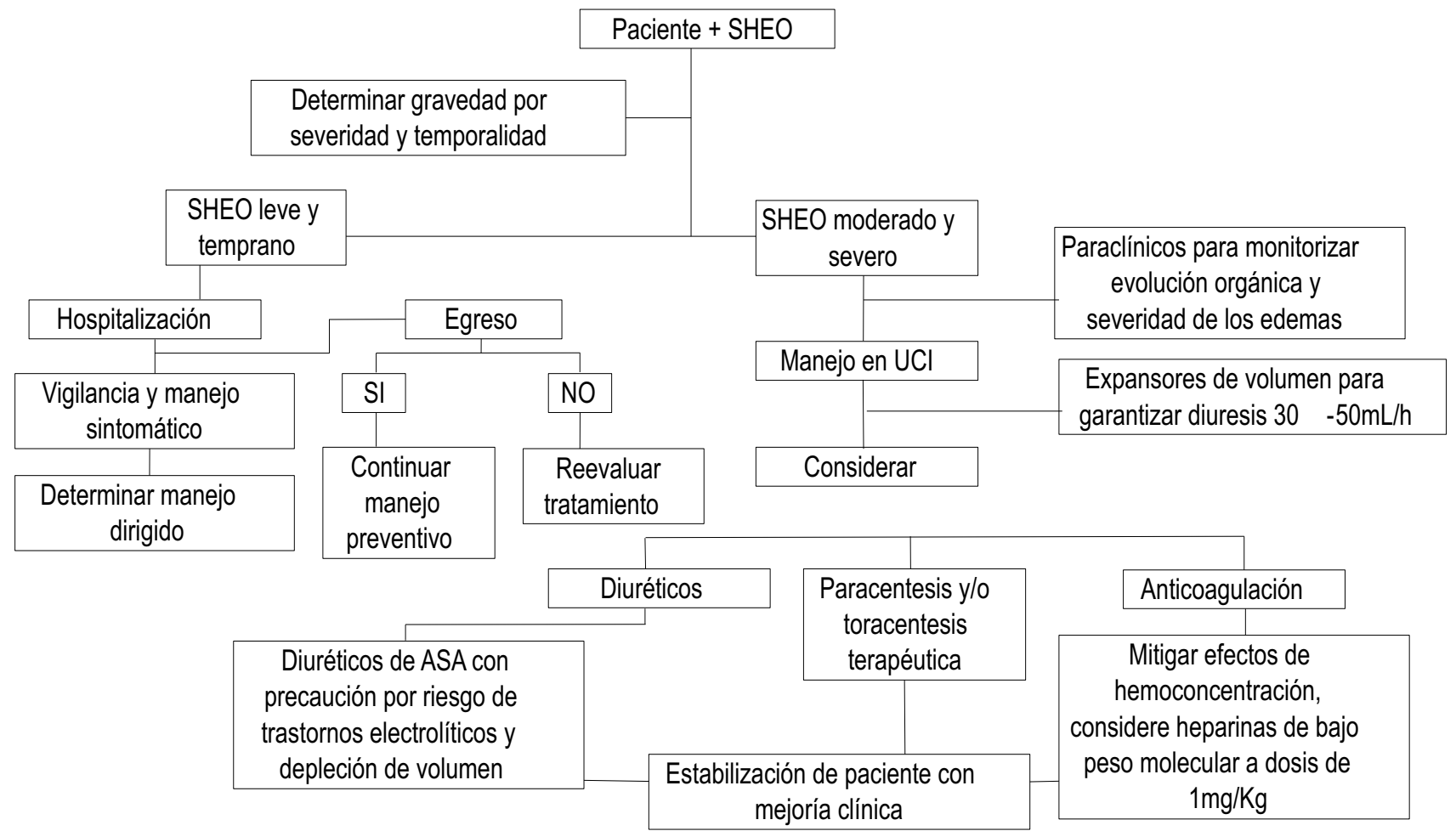

Fuente: Elaboración de los autores.

Figura 2. Algoritmo de manejo de pacientes con SHEO 
presentan pueden ser moderados o severos. El pilar fundamental en el tratamiento es resolver de manera eficaz el estado hipovolémico hiponatremico, con el objetivo de mantener la homeostasis de los demás sistemas orgánicos, siendo el riñón el principal órgano afectado. Por tanto, es importante evaluar siempre una estrategia preventiva una vez el cuadro se encuentre en una fase de convalecencia, sustentado en la evidencia a la fecha, que conduzca a evitar la aparición del SHEO especialmente con la aplicación de las medidas de identificación de los pacientes de alto riesgo, con la estimulando en forma personalizada, evitando la aplicación de HCG en estos pacientes e indicando la medicación y pasos adecuados pos aspiración folicular.

\section{Responsabilidades éticas}

Protección de personas y animales. Los autores declaran que para esta investigación no se han realizado experimentos en seres humanos ni en animales.

Confidencialidad de los datos. Los autores declaran que han seguido los protocolos de su centro de trabajo sobre la publicación de datos de pacientes.

Derecho a la privacidad y consentimiento informado. Los autores han obtenido el consentimiento informado del paciente referido en el artículo. Este documento obra en poder del autor de correspondencia.

\section{Conflicto de intereses}

Los autores declaran no tener conflicto de interés.

\section{Referencias}

1. Serrano J, Meijide H, Porteiro J, Asensio P. Derrame pleural y ascitis después de tratamiento con gonadotrofina coriónica humana. Galicia Clin. 2014; 75(3):133-5.

2. Mai Q, Hu X, Yang G, Luo Y, Huang K, Yuan Y, et al. Effect of letrozole on moderate and severe early-onset ovarian hyperstimulation syndrome in high-risk women: a prospective randomized trial. Am J Obstet Gynecol. 2017; 216(1):42.e1-42.e10. Disponible en: https://doi.org/ 10.1016/j.ajog.2016.08.018

3. Poyo A. Plan de cuidados de Enfermería en el Síndrome de Hiperestimulación Ovárica. Rev enferm CyL. 2016; 8(2):10-7.

4. Corbett S, Shmorgun D, Claman P, Cheung A, Sierra S, Carranza B, et al. The Prevention of Ovarian Hyperstimulation Syndrome. J Obstet Gynaecol Canada. 2014; 36(11):1024-33. Disponible en: https://doi.org/ 10.1016/S1701-2163(15)30417-5

5. Cervantes E, Vallejo V, Luna R, Sandler B. Síndrome de hiperestimulación ovárica. Actualización, ventajas de su diagnóstico y tratamiento oportuno. Rev Mex Med la Reprod. 2014; 6(3):158-68.

6. Fatemi H, Popovic B, Humaidan P, Kol S, Banker M,
Devroey $\mathrm{P}$, et al. Severe ovarian hyperstimulation syndrome after gonadotropin-releasing hormone $(\mathrm{GnRH})$ agonist trigger and "freeze-all" approach in $\mathrm{GnRH}$ antagonist protocol. Fertil Steril. 2014; 101(4):1008-11. Disponible en: https:// doi.org/10.1016/j.fertnstert.2014.01.019

7. Nieto J, Zuluaga M, Aristizábal A, Serna L, Ocampo C, Zuluaga G. Falla renal aguda asociada a síndrome de hiperestimulación ovárica. Acta Med Colomb. 2016; 41(1):58-61.

8. Pfeifer S, Butts S, Dumesic D, Fossum G, Gracia C, La Barbera A, et al. Prevention and treatment of moderate and severe ovarian hyperstimulation syndrome: a guideline. Fertil Steril. 2016; 106(7):1634-47. Disponible en: https://doi.org/10.1016/j.fertnstert.2016.08.048.

9. American Society for Reproductive Medicine, Birmingham. Ovarian hyperstimulation syndrome. Fertil Steril. 2008; 90(5):S188-93. Disponible en: https:// doi.org/10.1016/j.fertnstert.2008.08.034.

10. Pooniya S, Behera C, Mridha A, Bhardwaj D, Millo T. Fatal ovarian hyperstimulation syndrome in an anonymous egg donor. Med Leg J. 2016; 84(4):219-23. Disponible en: https://doi.org/10.1177/0025817216665074.

11. Shields R, Vollenhoven B, Ahuja K, Talmor A. Ovarian hyperstimulation syndrome: A case control study investigating risk factors. Aust New Zeal J Obstet Gynaecol. 2016; 56(6):624-7. Disponible en: https://doi.org/10.1111/ajo.12515.

12. Miller I, Chuderland D, Grossman H, Ron-EI R, Ben-Ami I, Shalgi R. The Dual Role of PEDF in the Pathogenesis of OHSS: Negating Both Angiogenic and Inflammatory Pathways. J Clin Endocrinol Metab. 2016; 101(12): 4699-709. Disponible en: https://doi.org/10.1210/jc.20161744.

13. El Tokhy O, Kopeika J, El-Toukhy T. An update on the prevention of ovarian hyperstimulation syndrome. Women's Health. 2016; 12(5):496-503. Disponible en: https://doi.org/10.1177/1745505716664743.

14. Guo J, Zhang D-D, Zhao Y, Zhang D, Zhang X-M, Zhou C$Q$, et al. Pharmacologic Interventions in Preventing Ovarian Hyperstimulation Syndrome: A Systematic Review and Network Meta-Analysis. Sci Rep. 2016; 6:1-11. Disponible en: https://doi.org/10.1038/srep19093.

15. Kwik M, Maxwell E. Pathophysiology, treatment and prevention of ovarian hyperstimulation syndrome. Curr Opin Obstet Gynecol. 2016; 28(4):236-41. Disponible en: https://doi.org/10.1097/GCO.0000000000000284.

16. Youssef M, Mourad S. Volume expanders for the prevention of ovarian hyperstimulation syndrome. Cochrane Database Syst Rev. 2016;(8). Disponible en: https://doi.org/10.1002/14651858.CD001302.pub3.

17. Kollmann M, Martins W, Lima M, Craciunas L, Nastri C, Richardson A, et al. Strategies to improve the outcomes of assisted reproduction in women with polycystic ovarian syndrome: a systematic review and meta-analysis. Ultrasound Obstet Gynecol. 2016; 48(6):709-18. Disponible en: https://doi.org/10.1002/uog.15898.

18. Rose B. A new treatment to avoid severe ovarian hyperstimulation utilizing insights from in vitro maturation therapy. J Assist Reprod Genet. 2014; 31(2):195-8. Disponible en: https://doi.org/10.1007/s10815-013-0143-6.

19. Kasum M, Orešković S, Franulić D, Čehić E, Lila A, Vujić G, etAl. Current medical strategies in the prevention of ovarian hyperstimulation syndrome. Acta Clin Croat. 2017; 56(1):133-42. Disponible en: https://doi.org/10.20471/ acc.2017.56.01.19. 